\title{
HORNOS UTILIZADOS EN LA FABRICACIÓN DE ALEACIONES FERROSAS Y NO FERROSAS
}

\author{
Carlos Sebastián Calvo
}

\begin{abstract}
RESUMEN
La fusión de aleaciones metálicas se lleva a cabo en hornos de fusión. Por lo general se usan chatarras y elementos de aleación como carga de dichos hornos. En el presente artículo, se reportan algunos de los más comúnmente utilizados hornos de fusión, tales como los diferentes tipos de hornos de crisol, hornos reverberos, hornos de cubilote y los hornos eléctricos de arco y de inducción. Los hornos de fusión utilizados en la industria de fundición son fabricados en diversas configuraciones. La selección de la unidad de fusión es una de las más importantes decisiones que deben tomar las empresas de fundición, teniendo en cuenta varios factores de importancia. El diseño del horno es un proceso complejo y puede ser optimizado considerando varios factores.
\end{abstract}

PALABRAS CLAVE: Fusión, fundición, horno, aleaciones ferrosas, aleaciones no ferrosas, crisol, cubilote, horno de hogar abierto, horno rotativo, inducción, arco eléctrico.

\section{MELTING FURNACES USED IN THE MANUFACTURE OF FERROUS AND NON-FERROUS ALLOYS}

\begin{abstract}
Melting alloys are manufactured in melting furnaces. Scraps and alloy elements are used to charge the furnace. Some of the more commonly used melting furnaces such as crucible furnaces, reberbatory furnaces, cupola furnaces, rotary furnaces, induction furnaces, and electric arc furnaces, are studied here. Melting furnaces used in the foundry industry are made of many diverse configurations. The selection of the melting unit is one of the most important decisions that foundries should make, taking into consideration several important factors. Furnace design is a complex process, and the design can be optimized on the base of multiple factors.
\end{abstract}

KeYwords: Melting, Foundry, Furnace, Ferrous alloys, Non-ferrous alloys, Crucible, Cupola, Reberbarory furnace, rotary furnace, Induction, Electric arc. 


\section{Introducción}

e describirán las variadas unidades de fusión utilizadas para la obtención del material metálico en estado líquido con el objetivo de producir piezas fundidas. El cuadro siguiente presenta las principales características de cada unidad.

HORNOS DE FUSIÓN PARA DIFERENTES APLICACIONES

\begin{tabular}{|c|c|c|c|}
\hline Fuente de energía & $\begin{array}{l}\text { Forma de la fuente de } \\
\text { energía }\end{array}$ & Aleaciones & Horno \\
\hline \multirow[t]{2}{*}{ Carbón } & Bituminoso en polvo & Fierros Fundidos & Llama directa \\
\hline & Antracita & Fierro Fundido & Cubilote \\
\hline Coque & Tamaño medio & $\begin{array}{l}\text { Fierros Fundidos } \\
\text { No Ferrosos }\end{array}$ & $\begin{array}{l}\text { Cubilote } \\
\text { Horno de Crisol }\end{array}$ \\
\hline Petróleo & $\begin{array}{l}\text { Diesel o Residual } \\
\text { Diesel o Residual }\end{array}$ & $\begin{array}{l}\text { No ferrosos } \\
\text { Hierro, Acero }\end{array}$ & $\begin{array}{l}\text { Horno de Crisol } \\
\text { Horno de Hogar } \\
\text { Abierto }\end{array}$ \\
\hline Gas & $\begin{array}{l}\text { Gas licuado de } \\
\text { Petróleo o Gas Natural } \\
\text { Licuado } \\
\text { Gas licuado de } \\
\text { Petróleo o Gas Natural } \\
\text { Licuado }\end{array}$ & $\begin{array}{l}\text { No ferrosos } \\
\text { Hierro, Acero }\end{array}$ & $\begin{array}{l}\text { Horno de Crisol } \\
\text { Horno de Hogar } \\
\text { Abierto }\end{array}$ \\
\hline \multirow[t]{4}{*}{ Electricidad } & Arco Directo & $\begin{array}{l}\text { Acero, Fierros } \\
\text { Fundidos }\end{array}$ & $\begin{array}{l}\text { Horno de Arco } \\
\text { eléctrico de una, } \\
\text { dos o tres fases }\end{array}$ \\
\hline & Arco Indirecto & $\begin{array}{l}\text { No Ferrosos, Fierros } \\
\text { Fundidos }\end{array}$ & $\begin{array}{l}\text { Horno de arco } \\
\text { Indirecto }\end{array}$ \\
\hline & Resistencia & No Ferrosos & $\begin{array}{l}\text { Horno de resistencia } \\
\text { central irradiante }\end{array}$ \\
\hline & Inducción & Todos los metales & $\begin{array}{l}\text { Horno de Inducción } \\
\text { Basculante }\end{array}$ \\
\hline
\end{tabular}

Debido a lo requerimientos de fabricación, cualquier tipo de horno puede ser el recomendable para una particular operación. La elección puede ser dictada por consideraciones de costo inicial, costo relativo promedio de mantenimiento y reparación, costo base de operación, disponibilidad y costos relativos de las fuentes de energía en una localidad en particular, condiciones ambientales y nivel de ruido en operación, eficiencia de fusión (particularmente velocidad de fusión), grado de control de composición química del metal, temperatura de fusión del material metálico, y experticia del personal. 


\section{Principales hornos de fusión utilizados en la fabricación de aleaciones no ferrosas}

\subsection{Hornos de crisol}

Los hornos de crisol (figura 1), son usados ampliamente para la fundición en lotes de aleaciones no ferrosas como aquellas de aluminio, cobre, zinc y magnesio. En un horno de crisol, el metal fundido es mantenido en una estructura con forma de marmita (crisol). Los calentadores generalmente a combustible fuera de esta estructura, generan el calor que pasa a través del crisol hasta el metal fundido. En muchas operaciones de fundición, se acumula escoria en el revestimiento de la superficie metálica, mientras que en el fondo se acumulan lodo pesado no fundido. Ambos reducen la vida útil del crisol y deben ser retirados para ser reciclados o tratados como residuos.

El proceso de fundir los metales en crisol es uno de los más antiguos y sencillos. Se emplea todavía mucho en la fundiciones modernas, y probablemente se seguirá usando porque el costo inicial es barato y el metal se funde fuera del contacto con el combustible.

Los hornos de crisol suelen dividirse en tres clases, según el procedimiento empleado para colar el caldo contenido en los crisoles (figura 2). En los hornos de crisol propiamente dichos, los crisoles están totalmente dentro de la cámara del horno y se extraen de ella para tratar el metal. (a) En los hornos de crisol fijo no basculantes (hornos estáticos de crisol fijo) existe un solo crisol fijo al horno que sobresale de la cámara de calefacción, (b) por lo que los gases de combustión no pueden tener ningún contacto con el caldo y como no es posible bascularlos para colar, su contenido de caldo solo puede pasarse a los moldes sacándolo del crisol del horno con una cuchara. Por otro lado, los hornos basculantes de crisol fijo son análogos a los anteriores, pero toda la estructura del horno

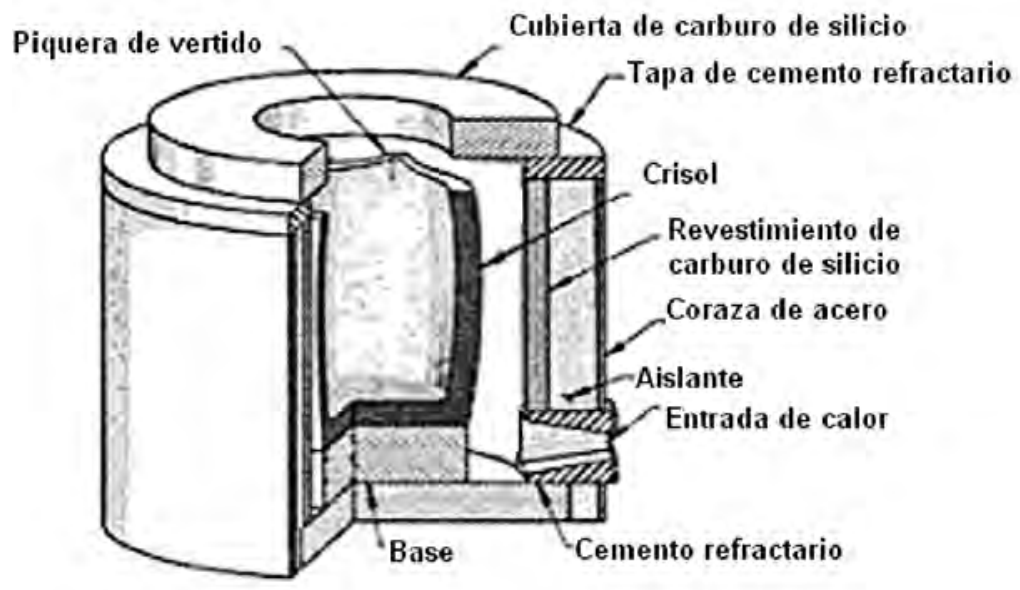

Fig. 1 Horno de crisol 


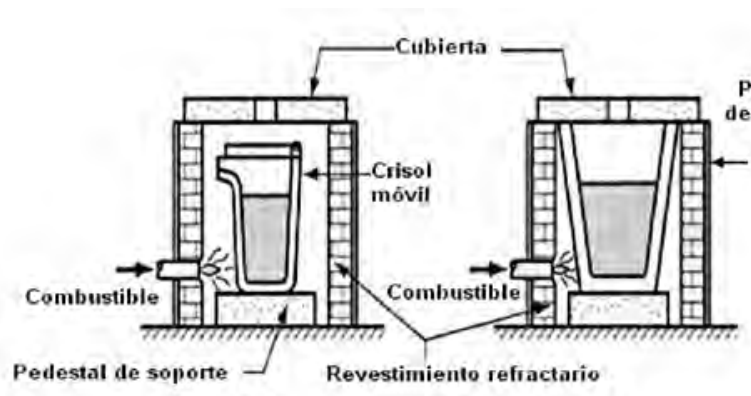

(a)

(b)

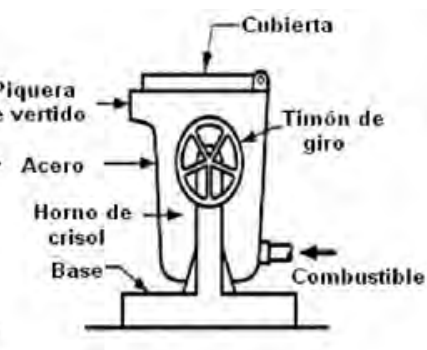

(c)

Fig. 2. Tres tipos de hornos de crisol: a) Crisol móvil, b) Crisol estacionario o fijo, y c) Crisol basculante

puede inclinarse para colar el caldo por vertido en cucharas o directamente a los moldes; el eje de rotación del horno puede ser central o transversal a la piquera de colada y situado precisamente en la piquera; en este último caso el contenido del crisol del horno se vierte íntegramente en la cuchara.

Los hornos de crisol pueden ser hornos de foso, hornos a nivel del suelo o bien hornos levantados respecto al suelo (figura 3). El tipo de foso, suele calentarse por coque que se carga alrededor y por encima de los crisoles (que se sierran con una tapa de refractario) para producir la fusión y el sobrecalentamiento sin necesidad de cargar más coque. El combustible descansa sobre una parrilla bajo la cual hay un cenicero y foso de cenizas. El tiro puede ser natural o forzado, es decir, producido por una chimenea o mediante un pequeño ventilador que trabaja a presiones de 50 a $75 \mathrm{~mm} \mathrm{H}_{2} \mathrm{O}$. El último método es preferible para controlar mejor el calor y la atmósfera del horno.

Los hornos calentados por gas o petróleo diesel o residual, son más fáciles de controlar y funden más rápidamente que los otros, pero imponen condiciones más duras a los crisoles y los refractarios. Los crisoles son de capacidad variable, pueden contener hasta aproximadamente $160 \mathrm{Kg}$. de acero, aunque son más corrientes las capacidades de 49 a $90 \mathrm{Kg}$. para latones, la capacidad suele ser de $70 \mathrm{Kg}$. Los crisoles grandes exigen algún mecanismo de elevación que permita sacarlos del horno, mientras los más pequeños pueden ser manejados con tenazas por uno o dos hombres. En algunos casos se han usado en estos hornos crisoles de hasta $180 \mathrm{Kg}$. de capacidad; la ventaja que se les admite es que hay menos perturbaciones y menos salpicaduras del caldo cuando se le transfiere desde la unidad de fusión hasta los moldes.

Cuando se encienden los hornos de crisol basculantes se calienta el crisol vacío, al principio suavemente, con la menor llama posible que puedan dar los quemadores durante los primeros $10 \mathrm{~min}$. Después se aumenta por etapas la velocidad de calentamiento hasta, que el crisol se ponga al rojo, en cuyo momento se le carga y se pone el quemador al máximo. El tiempo necesario para llevar los crisoles al rojo debe ser de, aproximadamente 30 min. para capacidades de hasta $300 \mathrm{Kg}$. de latón, $45 \mathrm{~min}$. para 450 a $700 \mathrm{Kg}$. de latón o $225 \mathrm{Kg}$. de aluminio y de $75 \mathrm{~min}$. para $450 \mathrm{Kg}$. de aluminio. 
Los crisoles ${ }^{4}$ deben cargarse con el horno vertical, empleando tenazas suficientemente largas para que puedan llegar al fondo del crisol.

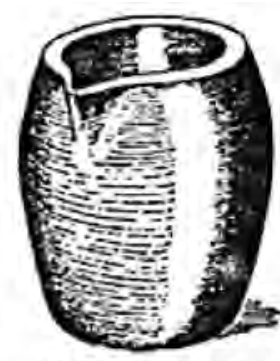

a. Crisol tipico

\section{b. Horno de} crisoles muiltiples, con tiraje natural

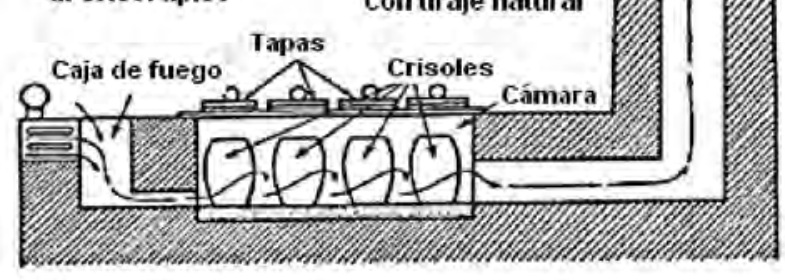

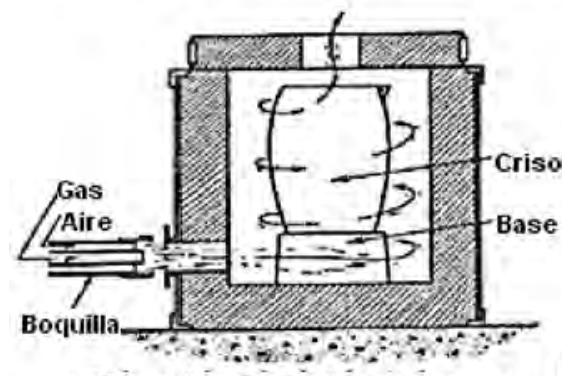

c. Horno de crisol calentado por gas d. Horno de crisol calentado por coque

Fig. 3 Crisol y hornos de crisol diversos

\subsection{Hornos de llama directa}

Un horno de llama directa es esencialmente un tipo de horno de hogar abierto. La unidad es calentada solamente en un extremo usando un combustible apropiado. El aire para la combustión puede ser precalentado en regeneradores como el caso del horno SiemensMartin. El combustible, generalmente carbón bituminoso, es pulverizado por un triturador rotativo y soplado al horno como polvo fino, aunque también se puede usar petróleo diesel o residual. Los productos de combustión y la llama pasan sobre la carga metálica y los gases son extraídos a través de una chimenea. Estos hornos ${ }^{4}$ son cargados con chatarras, arrabios y otros ingredientes, removiendo las secciones de arco en su bóveda como 
muestra la figura 4. Son usados para fusión de fierro, algunos para fundiciones grises o para fundición blanca y maleable.

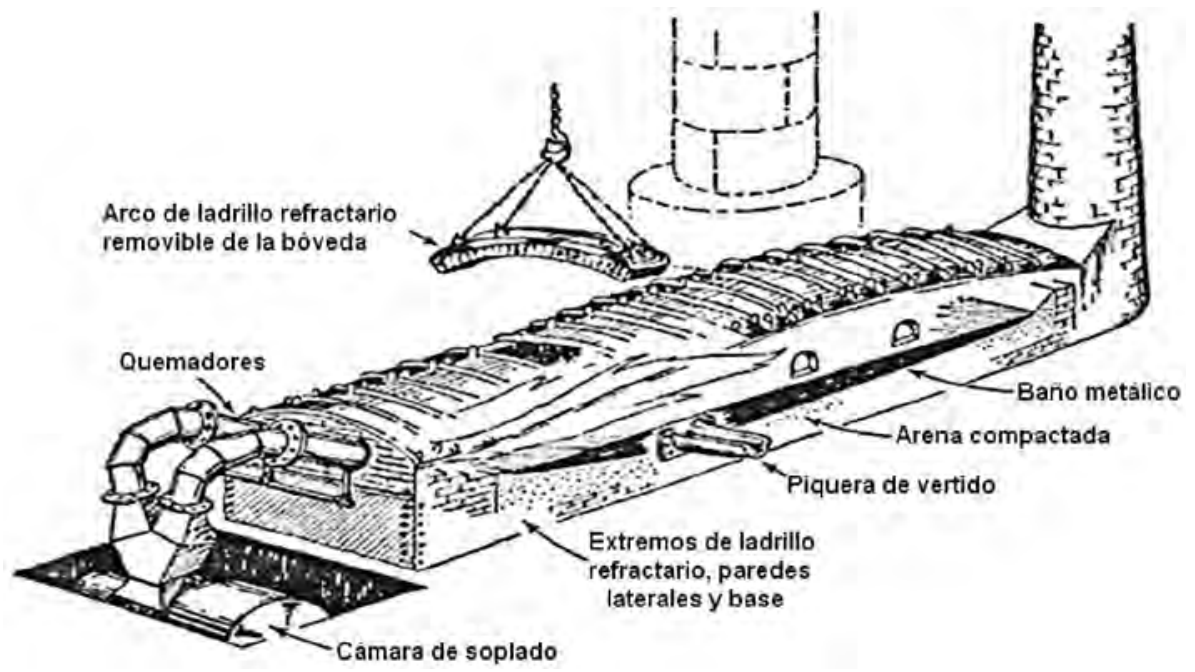

Fig. 4 Horno de llama directa ${ }^{4}$

\subsection{Hornos rotativos}

Un horno rotativo se compone de una envuelta cilíndrica de acero, revestido con material refractario, y que puede girar u oscilar lentamente alrededor de su eje principal. El horno suele terminar por sus extremos en troncos de cono; en uno de ellos está el quemador y en el otro la salida para los gases quemados, que frecuentemente pasan a un sistema de recuperación para precalentar el aire empleado en la combustión. El combustible puede ser gas, petróleo diesel o residual, o carbón pulverizado, y el aire se suministra mediante un ventilador o máquina soplante. En los hornos pequeños la rotación se puede dar a mano, pero la mayoría están montados sobre rodillos y se les hace girar por un dispositivo de cadena o de fricción. La elevada temperatura de la llama funde y sobrecalienta la carga y lleva una temperatura superior al refractario, que cede su calor a la superficie inferior del metal cuando al girar el horno se pone en contacto con ella. Este efecto acorta el tiempo de fusión y ayuda a salvar el efecto de aislante térmico den la capa de escoria. Se puede fundir en condiciones neutras, oxidantes o reductoras.

La capacidad de un horno rotatorio puede variar mucho. Para latones y bronces oscila entre unos $50 \mathrm{Kg}$. y 5 Ton. Y normalmente son de $50 \mathrm{Kg}$. a 2 Ton. Para la fundición de hierro, y en algunos casos acero, las capacidades pueden ser mucho mayores. El metal puede sangrarse por un agujero de colada único situado en la pared del cilindro, que se mantiene taponado con refractario mientras el horno gira. Las unidades grandes tienen un control para que el flujo sea uniforme durante la colada. En algunas instalaciones se pueden inclinar hacia delante o hacia atrás para facilitar la carga, la colada y el desescoriado. 
Los hornos rotativos sirven para una gran variedad de trabajos, incluyendo por un lado las fundiciones grises, de alta calidad o maleables y los aceros, y por otro cobre, bronce, aluminio y plomo.

Para las temperaturas más elevadas, mientras que el aceite, gas o carbón pulverizado dan resultados parecidos para temperaturas intermedias y bajas, por lo que la elección depende del precio y de la facilidad de suministro. El revestimiento de los hornos rotativos puede ser de material apisonado o de ladrillos refractarios de calidad, dependiendo el emplear uno o el otro revestimiento del trabajo que ha de realizarse en los hornos.

Los hornos basculantes semi-rotativos pueden tener capacidades de hasta 3 Ton. Para la fusión de cobre, bronce, latón y aluminio. Durante la fusión, y a intervalos, se les hace girar hacia un lado y hacia el contrario, lo que les distingue de los de rotación completa. Las temperaturas pueden llegar a $1300^{\circ} \mathrm{C}$. Una ventaja es que le horno ocupa poco espacio. Las figuras 5, 6 y 7 muestran diversas configuraciones de hornos rotativos ${ }^{4,8}$.

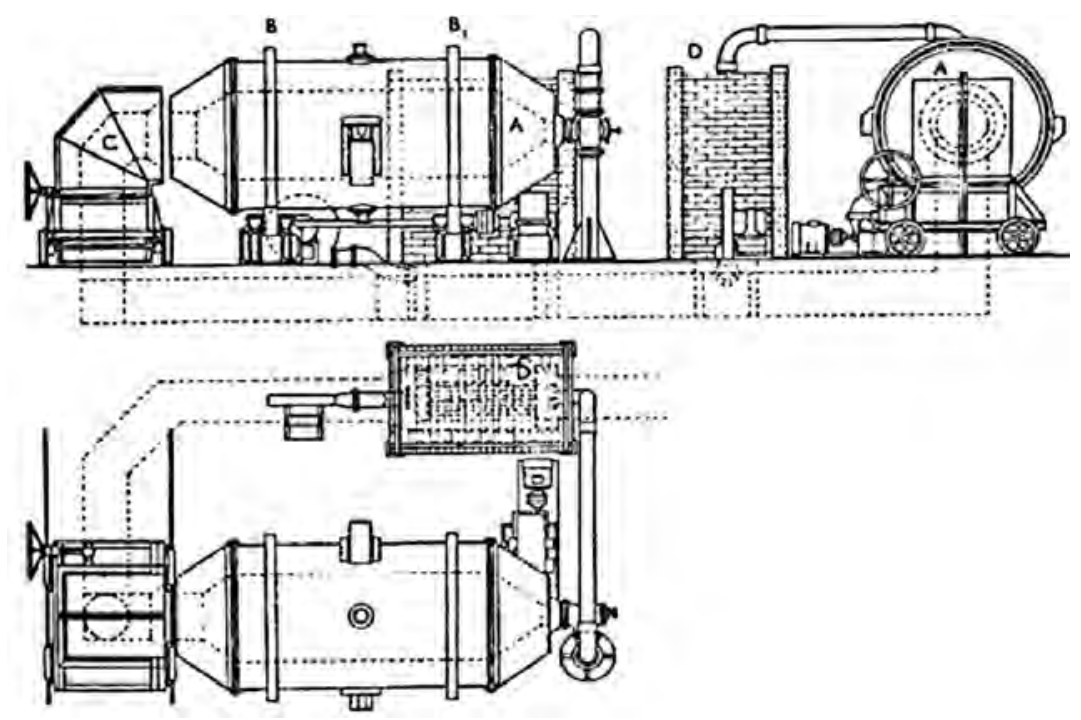

Fig. 5 Representación del diseño de la instalación de un horno rotativo ${ }^{4}$

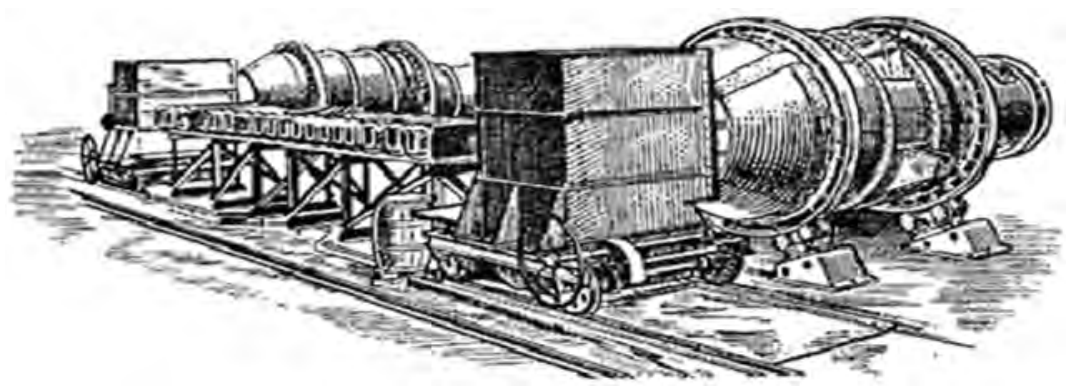

Fig. 6 Hornos rotativos a combustible pulverizado, mostrado cajas móviles de extracción de gases, los que se pueden usar para precalentamiento del aire. La carga se realiza en el extremo del lado de las cajas ${ }^{4}$ 


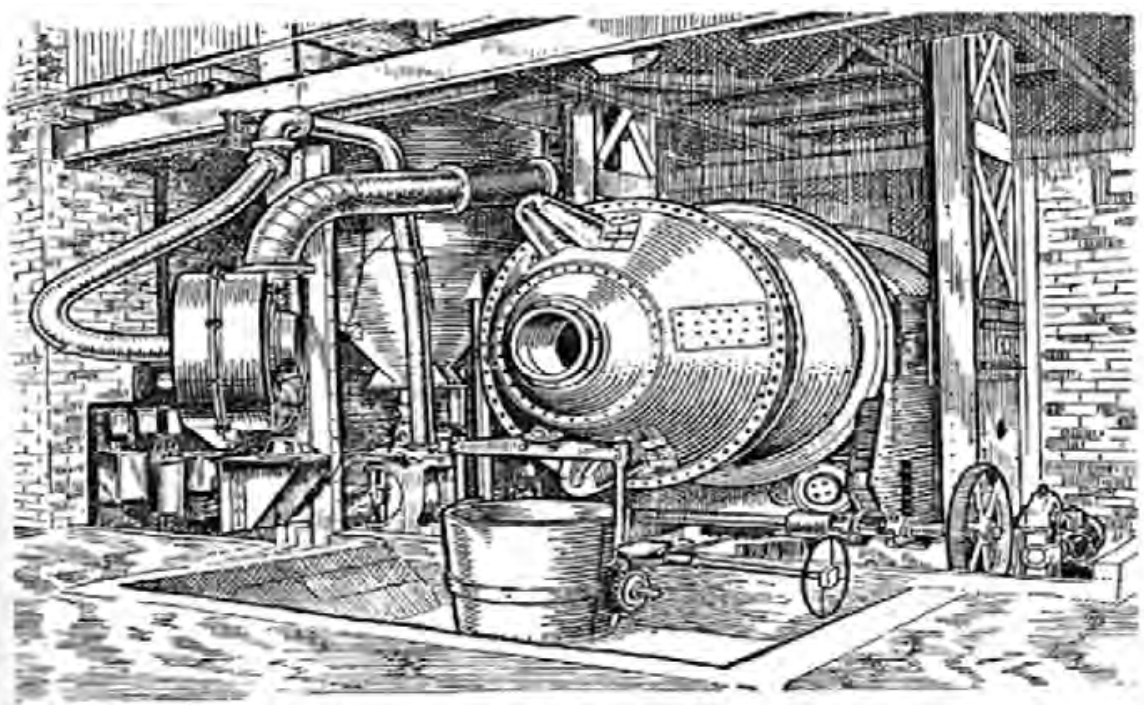

Fig. 7 Horno rotativo basculante según su eje longitudinal. El quemador esta a un lado para el vertido. El ángulo de basculamiento se puede controlar para permitir el vertido de pequeñas cantidades de metal fundido, de ser necesario ${ }^{4}$

\subsection{Horno eléctrico de resistencia central irradiante}

La fuente de calentamiento es una resistencia de grafito en forma de barra horizontal como se ilustra en la figura 8. El horno es cilíndrico y revestido internamente con material refractario 4 . La resistencia distribuye el calor irradiado permitiendo que el metal tome calor del material refractario. Este tipo de horno ha sido usado para fusión y sobrecalentamiento, especialmente de fierros fundidos pero también es apropiado para fusión de bronce y otras aleaciones de cobre.

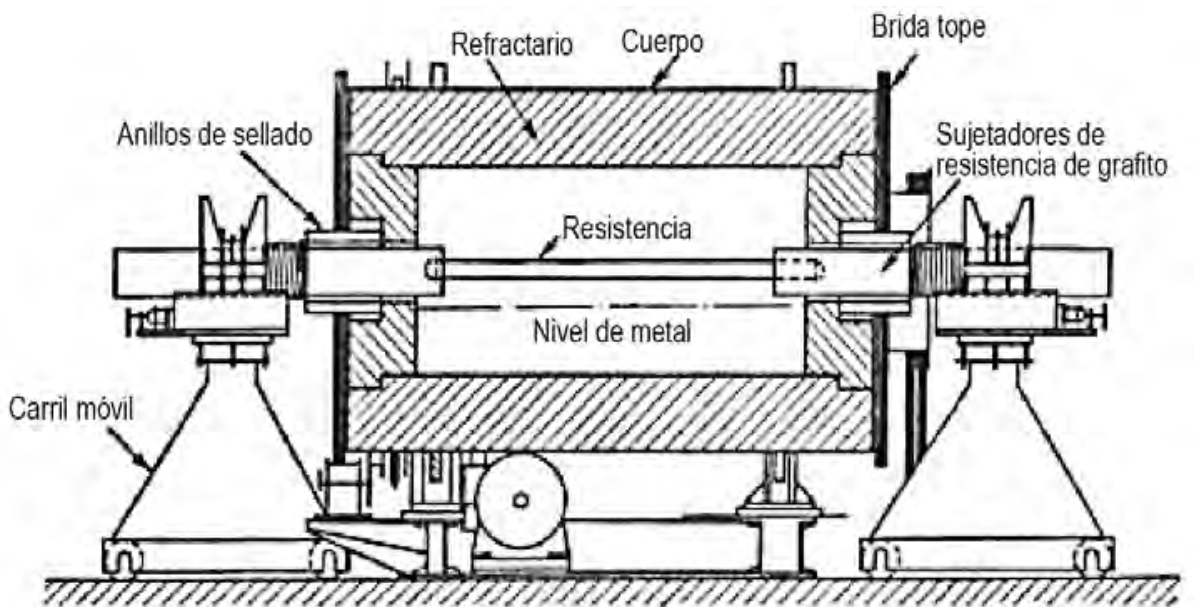

Fig. 8 Horno eléctrico de resistencia central irradiante $e^{4}$ 


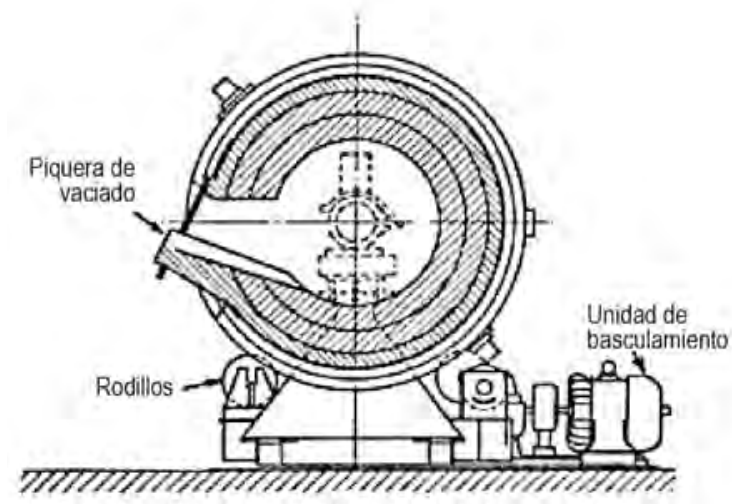

\section{Principales hornos de fusión para aleaciones ferrosas}

\subsection{Horno cubilote}

Utiliza el principio de combustión entre las materias primas para lograr la fusión`. Son utilizados comúnmente para las fundiciones grises y trabajan a temperaturas de hasta $1200^{\circ} \mathrm{C}$. Se cargan con metal (chatarra), coque y fundente. El cubilote es un horno de torre vertical, consiste en una en vuelta cilíndrica de acero que reposa verticalmente sobre una placa base sostenida usualmente por 4 columnas o vigas de acero (figura 9). La mayoría de los modernos se pueden abrir por el fondo, llevan compuertas centradas en la placa base que pueden abrirse hacia abajo después de un ciclo de funcionamiento para vaciar todos los residuos acumulados.
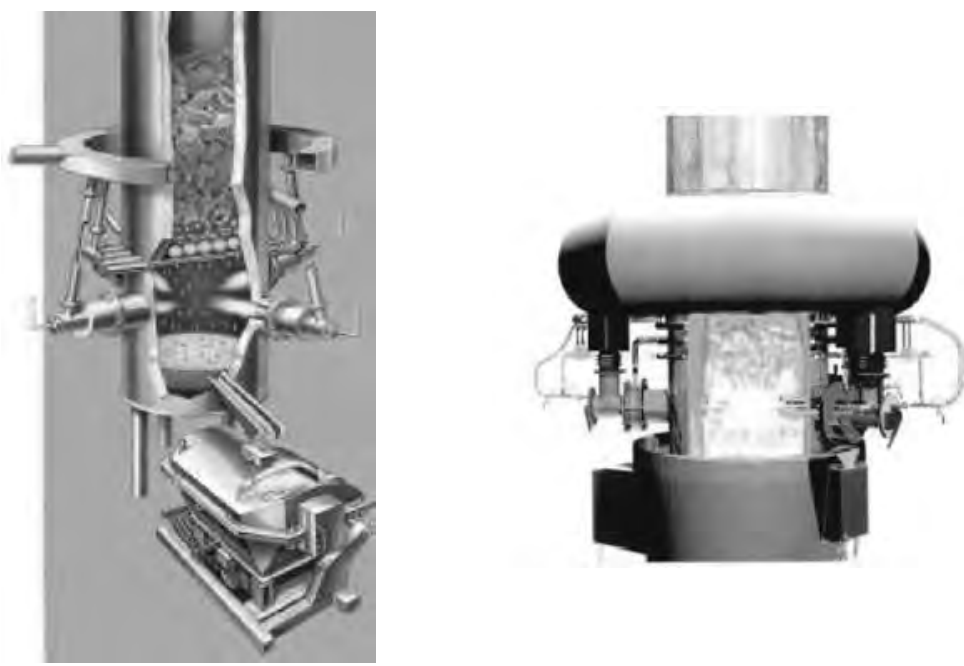

Fig. 9 Horno cubilote con anticrisol para tratamiento y ajuste de composición a la izquierda. A la derecha se detalla el sistema de insuflación de aire por toberas inyectorasc 
En la conducción correcta del cubilote se empieza por encender sobre el fondo y se añade coque en pequeñas cantidades hasta alcanzar el nivel de las toberas, el fuego asciende progresivamente hasta que todo el coque está caliente alimentado por tiro natural. Cuando el lecho está apunto se añaden las cargas para llenar el horno hasta el nivel del tragante. Se alternan las cargas de metal y coque, el fundente, que normalmente es caliza, se carga con el coque. Cuando el cubilote está lleno se comienza el soplado.

Mientras tanto se siguen añadiendo cargas de metal y coque para reemplazar al metal fundido y así se continúa hasta el fin de la colada. La escoria se sangra por el agujero correspondiente a intervalos de $45 \mathrm{a}$ 60 min. o siempre que se considere que se ha acumulado en el horno demasiada escoria.

En función de las características dimensionales del horno cubilote, se establecen las

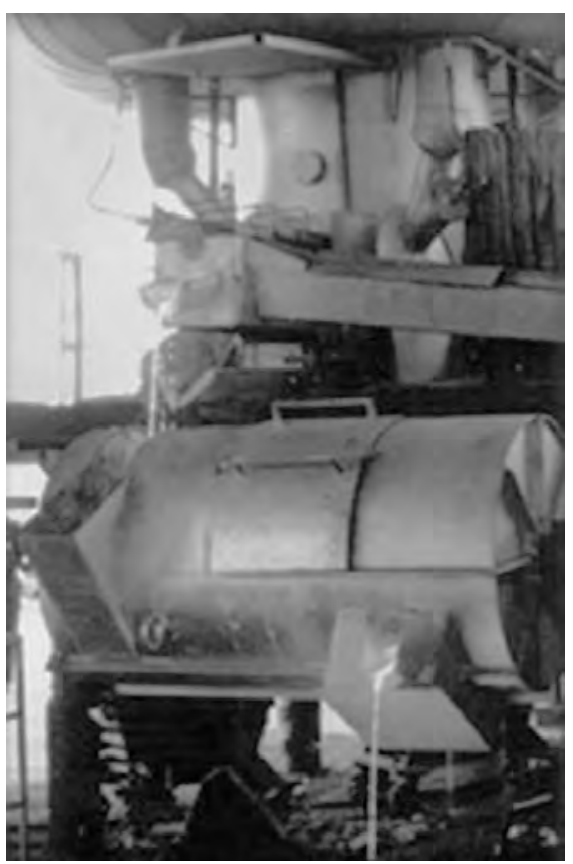

Fig.10 Anticrisol al pie del horno cubilote para ajuste y tratamientos en el estado líquido, en la elaboración del fierro fundido nodular variables de operación que a continuación se indican.

El cubilote con anticrisol ofrece la ventaja de obtener fundiciones de composición uniforme, exacta y dosificada según las necesidades. Facilita la desulfuración y eliminación de escoria. Evita que la fundición resulte contaminada por el coque del cubilote. Se adaptan a la fabricación de fundiciones de grafito esferoidal por adición de magnesio a la fundición líquida, tal como se muestra en la figura 10.

\subsection{Hornos eléctricos de arco directo}

Son usados principalmente por grandes fundiciones y plantas siderúrgicas. Se suministra calor mediante un arco eléctrico formado en base a tres electrodos de carbón o grafito. El horno es revestido con refractarios que se deterioran durante el proceso de fusión, lo que genera escoria. Se forman capas de escoria protectora en el horno mediante la adición intencional de sílice y cal. Puede añadirse fundentes como fluoruro de calcio para hacer que la escoria sea más fluido y más fácil de retirar. La escoria protege al metal derretido del aire y extrae ciertas impurezas.

Se añaden a la carga del horno residuos de metal, chatarras y retornos de fabricaciones anteriores y cal o piedra caliza. El equipo de recolección de polvo y humos controla las emisiones al aire del horno de arco eléctrico. 
El arco voltaico que se establece en los electrodos y la masa de fusión puede producir temperaturas de hasta $3500^{\circ} \mathrm{C}$. Con esto la chatarra de acero añadida al acero previamente refinado se funde y toda la carga del horno se libera ampliamente de las impurezas azufre, fósforo y carbono que se queman. El oxígeno necesario para la combustión se toma en parte del aire que está en contacto con e baño y en parte de las adiciones que lo contengan. Para terminar el proceso, se lleva el acero a la composición que se desea mediante adiciones.

Bajando los electrodos acorta el arco y aumenta la potencia, lo contrario ocurre al subirlos. El control se efectúa automáticamente. Casi todos los hornos empleados para la fusión son de tipo basculante. Los hornos grandes se cargan por arriba levantando la bóveda (estructura de ladrillos refractarios), el desplazamiento puede ser lateral, por puente grúa o giro alrededor de un pivote.

\subsection{Horno de arco indirecto}

El arco salta entre dos electrodos de carbón completamente separados e independientes de la carga, que se calienta solo por radiación. Consiste en una envoltura revestida con refractarios, con dos electrodos montados horizontalmente, el arco salta entre ellos en el centro de la cámara formada por el revestimiento refractario.

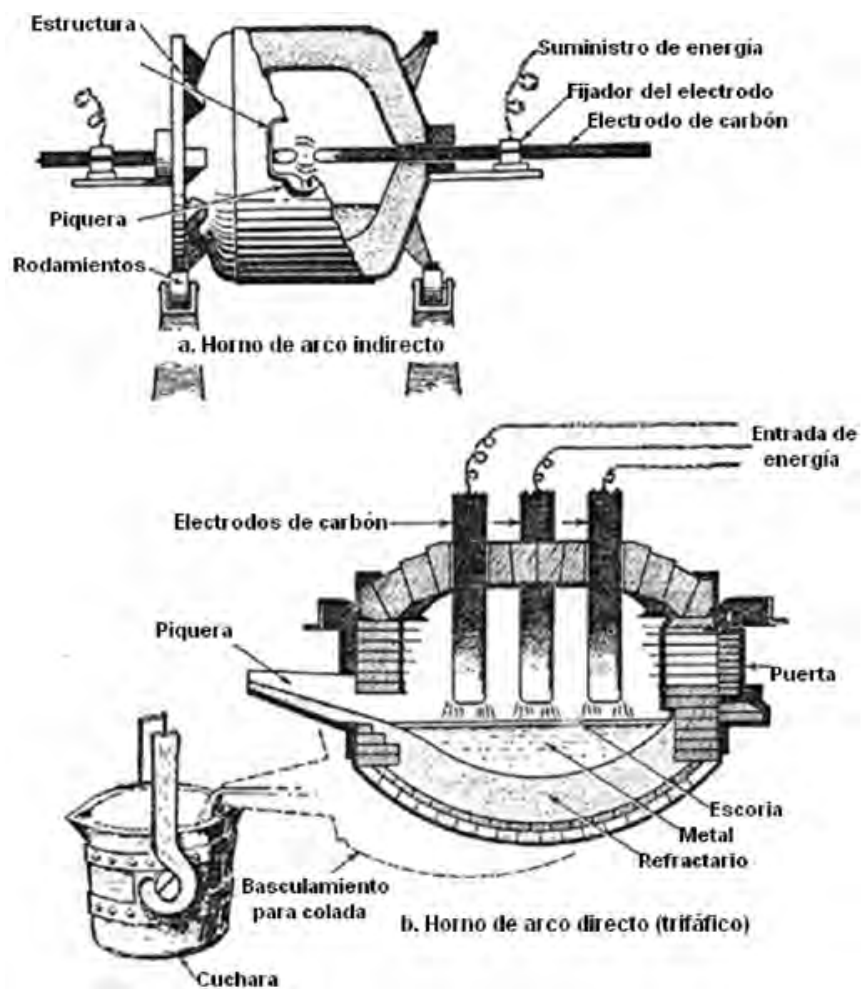

Fig. 11 Representación de la operación de los hornos de arco indirecto (a) y directo (b) 
El horno está montado sobre rodillos accionados por un motor que le da el balanceo. Se carga a través de la abertura de carga después de sacar los electrodos ${ }^{4}$.

\subsection{Horno eléctrico de inducción}

Funciona en base a un campo magnético, producido por la circulación de corriente alterna por las bobinas que envuelven el manto del horno. Este campo magnético cambiante produce que el metal se caliente y se funda.

Los hornos de inducción utilizan una corriente inducida para fundir la carga. La energía es del tipo de inducción sin núcleo dada por una corriente de alta frecuencia que suministra a la bobina primaria, enfriada por agua que circunda al crisol ${ }^{8}$ (figura 12).

Los hornos de inducción se basan en el principio físico según la cual los cuerpos metálicos sometidos a la acción de un campo magnético de corriente alterna se calientan tanto más cuanto más intenso es el campo magnético y cuanto más elevada es la frecuencia.

Están constituidos por una espiral cilíndrica (enfriada por circulación interior de agua) de tubo de cobre de sección rectangular o cuadrada, dentro de la cual va instalado un crisol que contiene el metal que se ha de fundir.

Por efecto del campo magnético generado por la espiral se induce una corriente a la masa metálica y la energía eléctrica absorbida se transforma en calor ${ }^{4}$. El crisol refractario no impide la acción del campo magnético, como se observa en los detalles de diseño de la figura 13.

Los hornos de inducción de alta frecuencia poseen notables ventajas: su producción es de gran calidad, con oxidaciones muy reducidas y análisis constantes. Sin embargo, los gastos de instalación son muy elevados. Se emplean particularmente en las fundiciones de aceros aleados especiales o de aleaciones de hierro colado y en menor escala en las fundiciones de hierro colado gris.
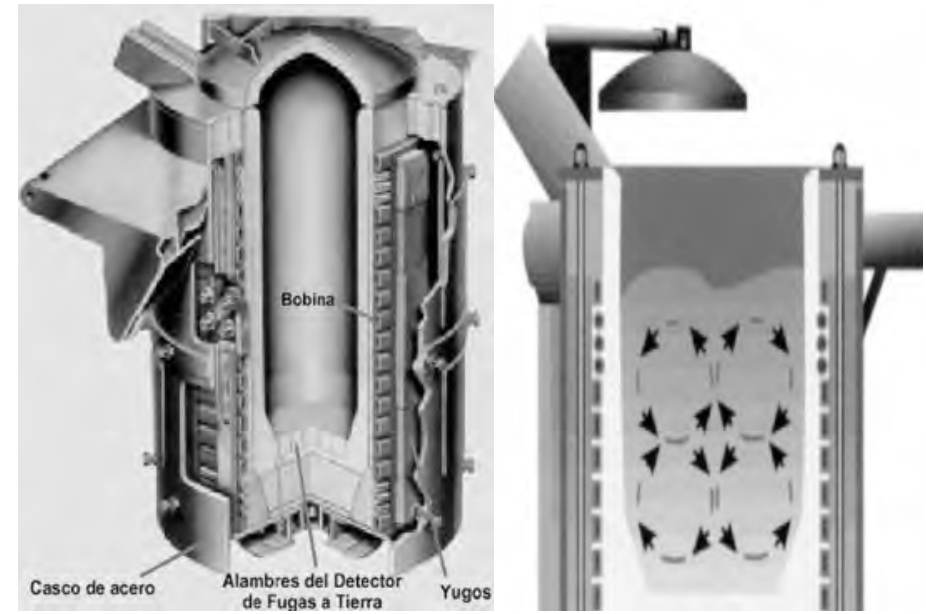

Fig. 12 Partes y principio de funcionamiento del horno de inducción ${ }^{8}$ 
Los hornos de inducción se han convertido gradualmente en los hornos más usados para la fundición ferrosa y, crecientemente, para aleaciones no ferrosas. Estos hornos tienen un excelente control metalúrgico y están relativamente libres de contaminación. Los hornos de inducción están disponibles en capacidades que van desde unas cuantas libras hasta 75 toneladas.

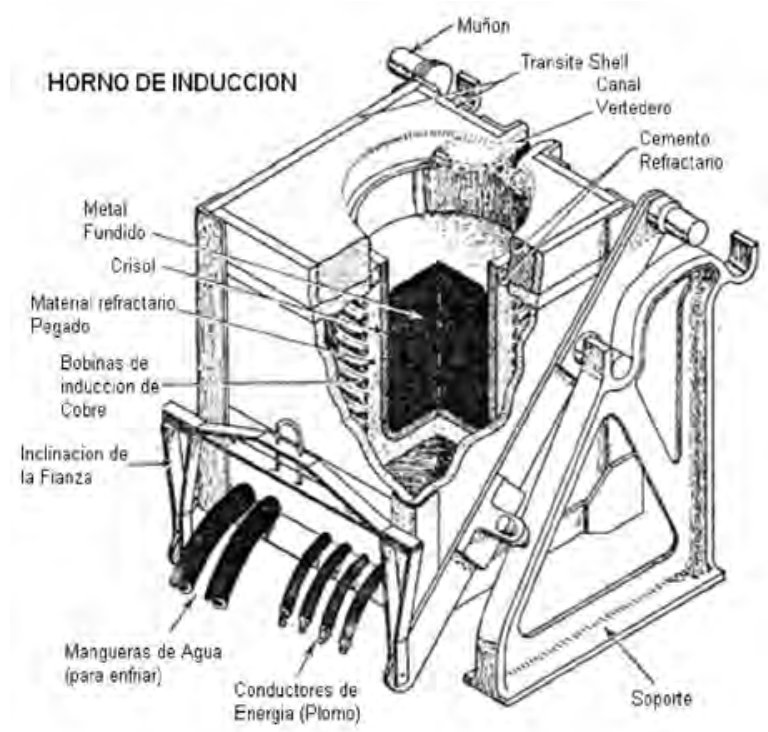

Fig. 13 Configuración del horno eléctrico de inducción y sus elementos auxiliares ${ }^{4}$

\subsubsection{Hornos de inducción a canal}

En la figura 14 se muestra una variante de este tipo de horno donde el canal secundario tiene forma de $V$ y el baño metálico principal esta encima de este. Gran parte del calor es generado en la sección en $V$, con rápida circulación del metal causada por efecto térmico y eléctrico. Es necesario en este caso mantener al menos un tercio de pie de baño para continuar añadiendo carga sólida. Este tipo de horno ha sido usado ampliamente para fusión de latones, de aleaciones de aluminio para colada en coquillas y de fierros fundidos.

\subsubsection{El horno de inducción con núcleo}

El horno de inducción con núcleo (figura 15) es la más eficiente de las unidades de fusión por inducción. La bobina de inducción esta esencialmente sumergida en el baño metálico. La inducción electromagnética bombea metal líquido a través de los canales alrededor del núcleo. Este horno requiere carga metálica liquida de arranque por lo que no es apropiado para producción intermitente. Se usa preferentemente para fusión de aleaciones no ferrosas donde se requieran largos periodos de operación continua. 


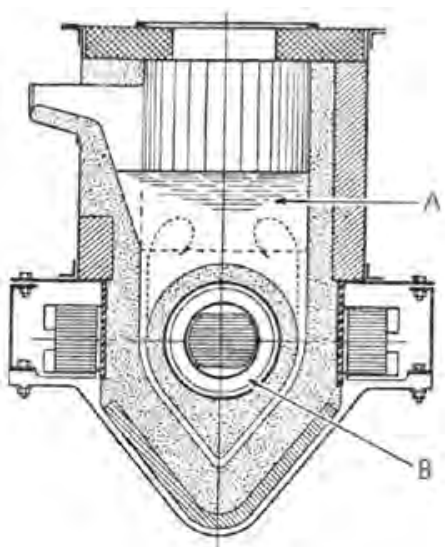

Fig. 14 Horno de inducción a canal tipo $V$, donde $A$ representa el baño metálico liquido y B es la bobina central ${ }^{4}$

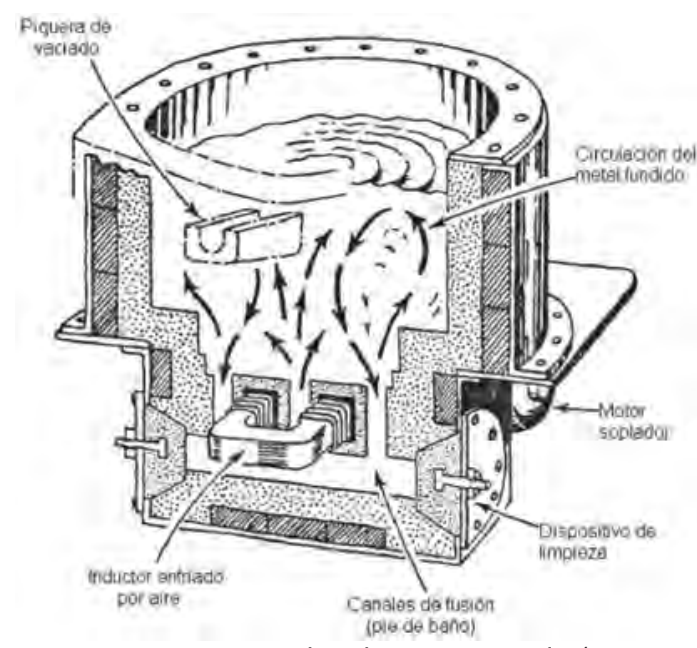

Fig. 15 Horno de inducción con núcleo ${ }^{4}$

\section{Criterios para la integración de los hornos de fusión en las unidades de produccion por fundición}

3.1 La figura 16 presenta el flujograma para el proceso de fundición. Una pieza encomendada es fabricada a partir de un modelo suministrado por el propio cliente o confec-

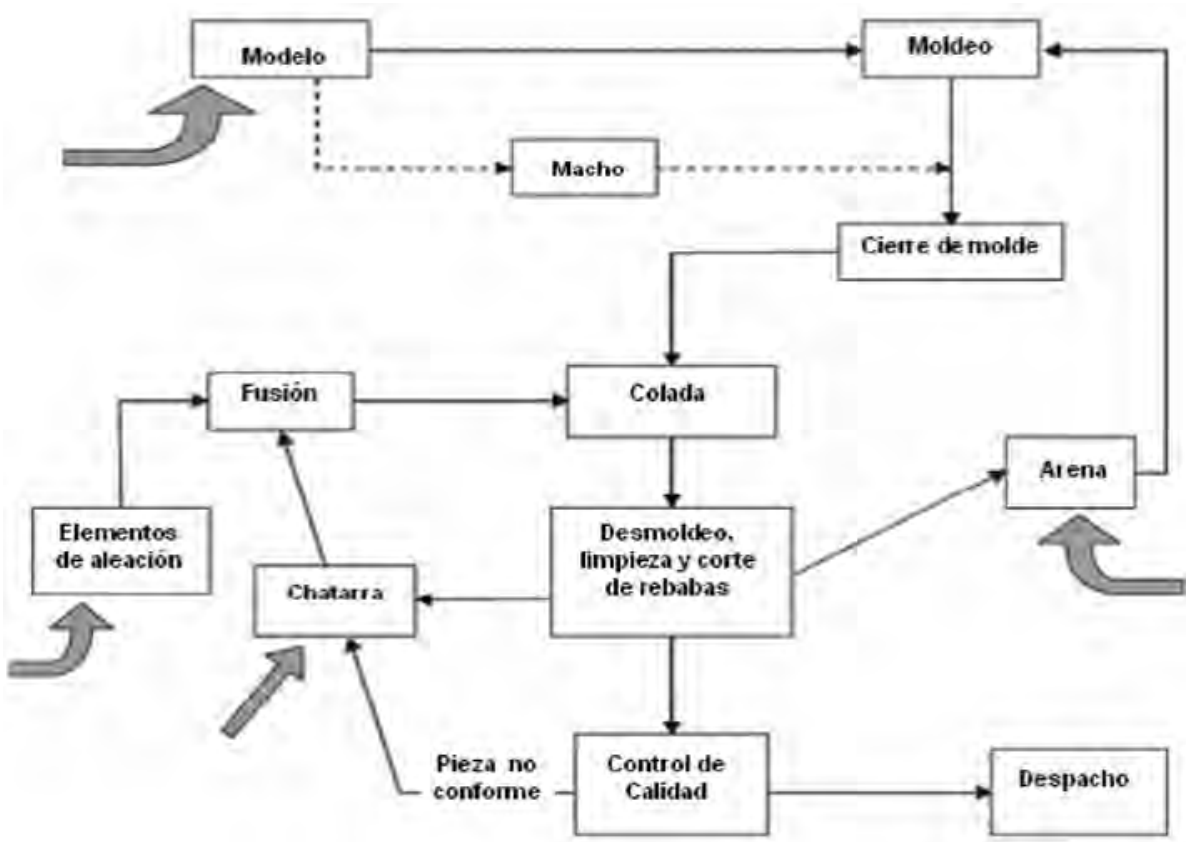

Fig. 16 Flujograma integrado de la producción de piezas por fundición 
cionada por terceros, o producida en la misma fundición. La capacidad productiva, en este caso, está limitada por el horno, pues es la fuente alimentadora de materia prima básica para el proceso.

3.2 La figura 17 ilustra un diagrama general de operaciones y procesos para el caso de la fundición en moldes de arena.

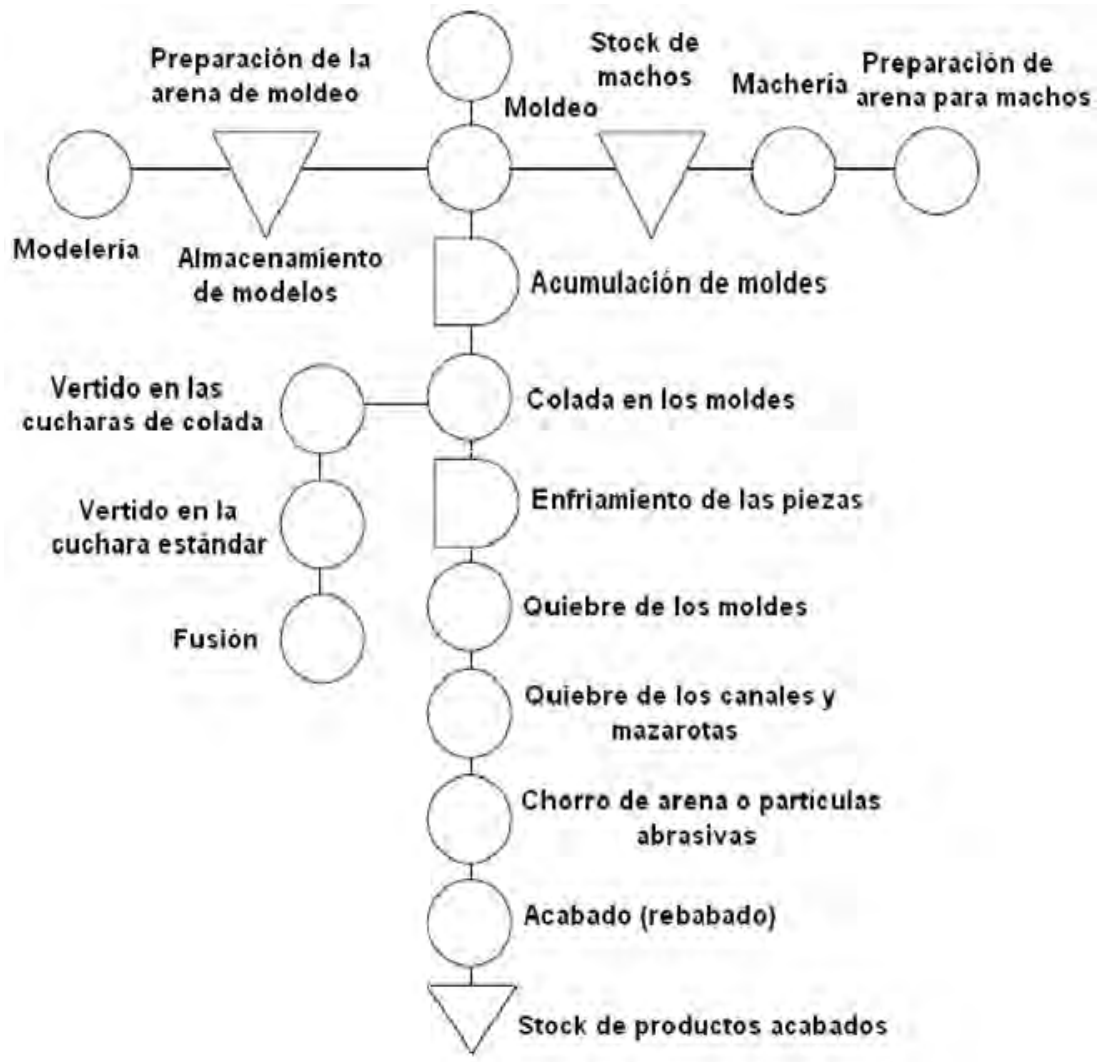

Fig. 17 Diagrama de operaciones y procesos de las operaciones de fundición en arena

3.3 La programación de la producción de los hornos en general es forzada por el sector de moldeo. A partir de la cartera de pedidos existente se genera una orden de fabricación para que el sector de moldeo construya los moldes. El sector de moldeo es proveedor del sector de fundición y juega un papel importante en la orden de producción. Puede haber un secuenciamiento de la construcción de los moldes de acuerdo con los tamaños de la pieza (piezas menores demoran más y son más difíciles de moldear), las aleaciones de las piezas (el margen de utilidad de aleaciones especiales es mayor que el de las otras), los plazos de entrega y los clientes preferenciales. Sin embargo, es común la aparición de stocks intermedios de moldes de arena para ser utilizados en la sección de fusión, subutilizando la capacidad de ese sector. 


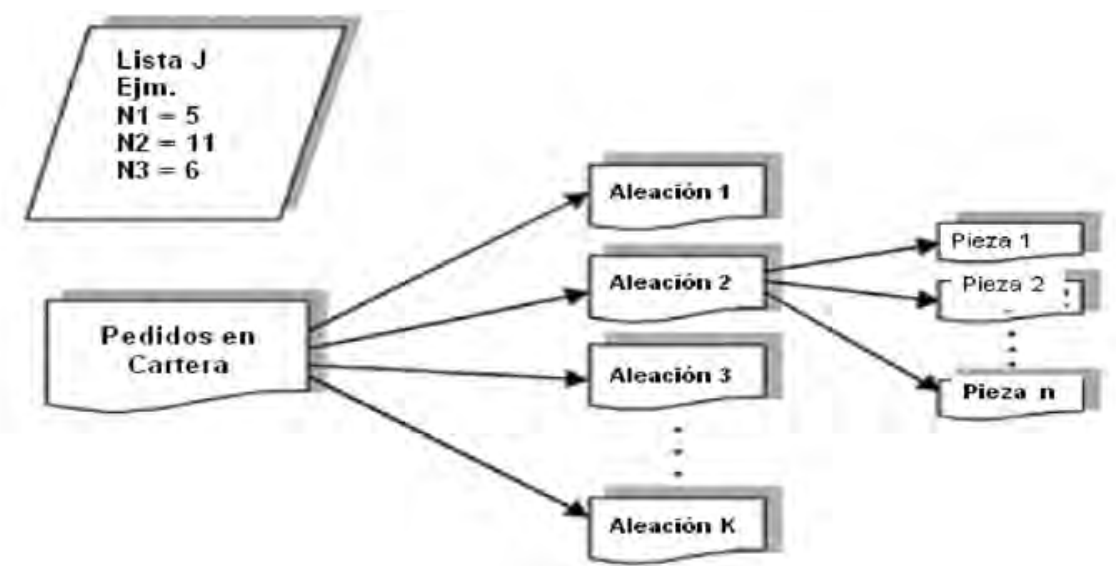

Fig. 18 Caracterización del problema, con fines de programación y control de la producción de una cartera múltiple de pedidos de productos de fundición

\section{Conclusiones}

3.1 Es posible integrar las diferentes unidades de producción en la industria de fundición oderna. La figura 19 presenta una posible integración de dichas unidades de una fundición que utiliza líneas de preparación de arena para moldes y machos, líneas de moldeo, fusión en diferentes hornos, colada desmoldeo, limpieza y maquinado, entre otras.

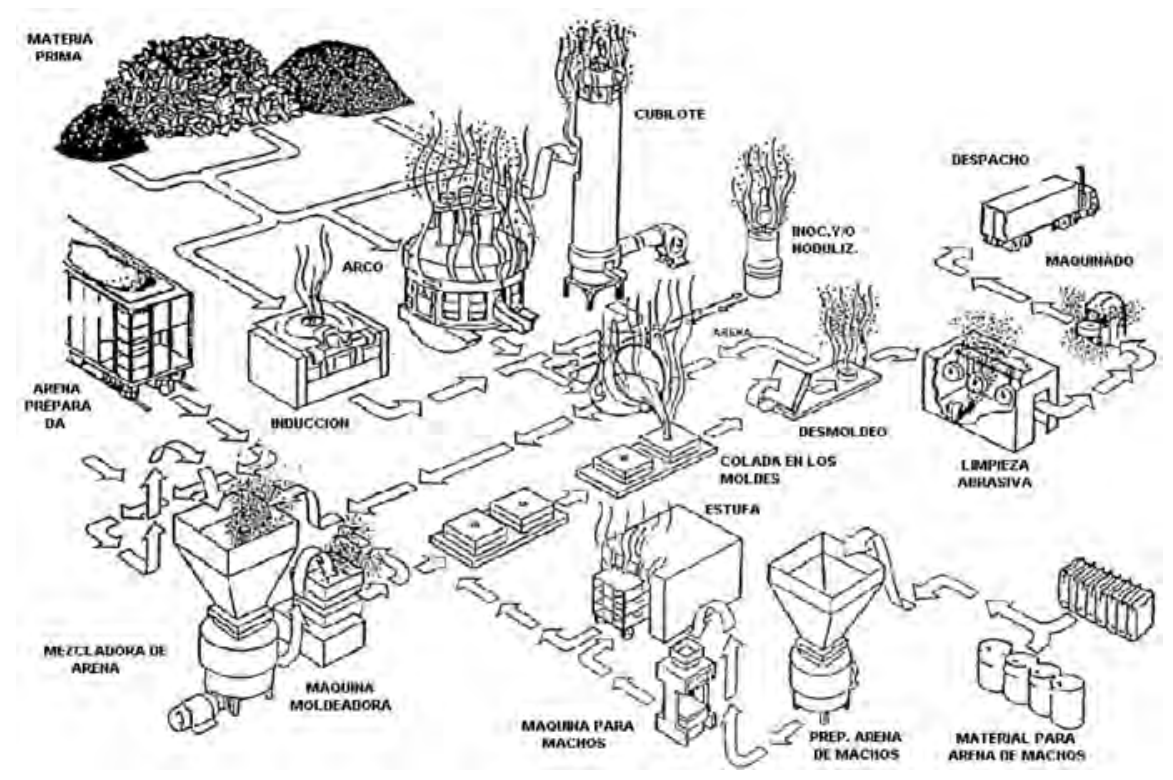

Fig. 19 Propuesta de una secuencia de operaciones integradas de una fundición que utiliza moldes de arena 
3.2 Problemas tales como restricciones de análisis químico a través de rangos ajustables, rendimientos diferenciados por materia prima y/o elementos químicos, fijaciones $\mathrm{y} / 0$ limitaciones de componentes, ajuste de cargas preliminares en el horno utilizado y análisis de costo marginal de materias primas, pueden ser resueltos rápidamente con ayuda de la gestión de las operaciones concentrada en el cálculo de cargas a un mismo horno de fusión, para la fabricación de una cartera múltiple de pedidos, según se ha esquematizado en la figura 18.

3.3 Es recomendable programar las operaciones de producción de lotes de piezas de diferente geometría en una misma jornada de fusión para diferentes grupos de aleaciones a elaborar. Es conveniente adecuar a cada caso, un diagrama de operaciones y procesos particular como el mostrado en la figura 20.

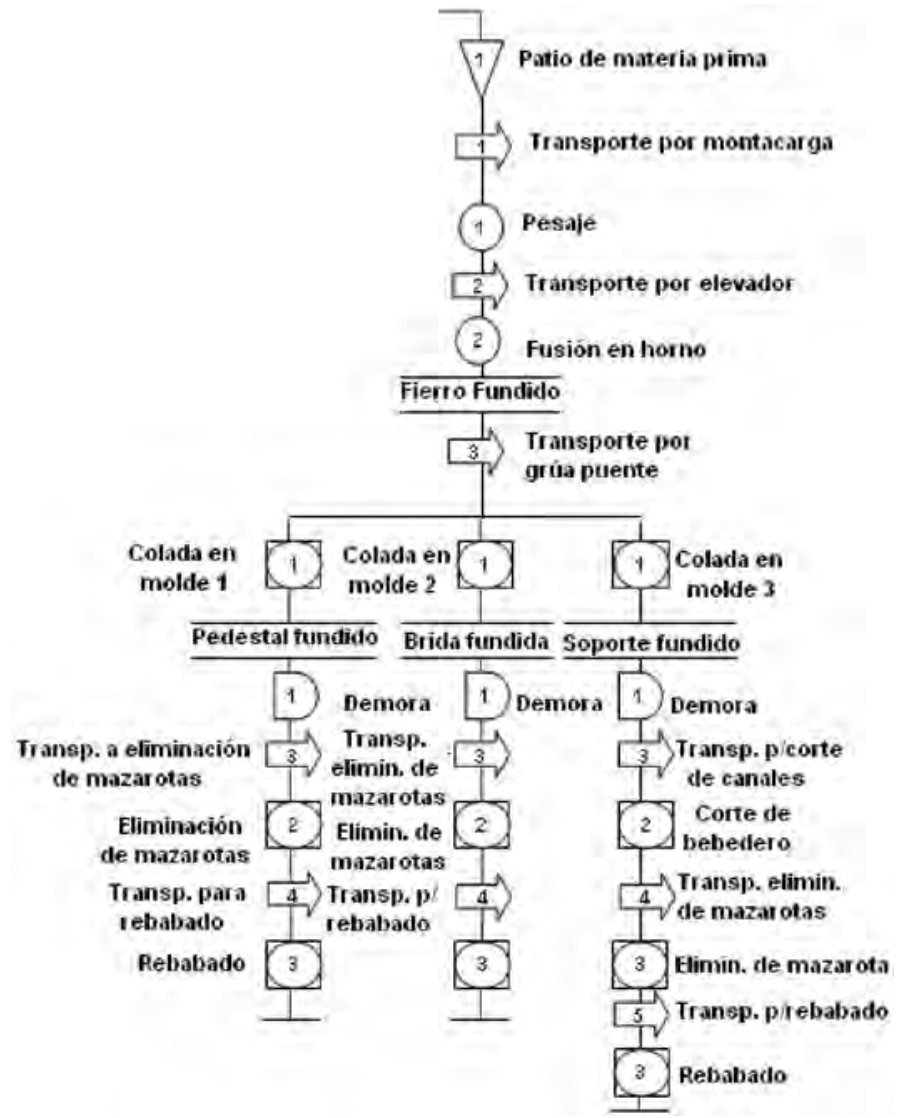

Fig. 20 Diagrama de operaciones y procesos de una jornada de fusión de diversos lotes de diferentes piezas en fierro fundido

3.3 Es posible utilizar modelos de programación lineal para optimizar la producción de los hornos de fundición. Las funciones matemáticas que aparecen tanto en la función objetivo como en las restricciones, son funciones lineales. De esta manera se cons- 
truirán modelos específicos de programación lineal que se ajustan a diversos tipos de problemas, como determinar:

a) La mezcla de productos que maximiza la ganancia en la operación de fusión en los diferentes hornos de fundición.

b) La combinación de métodos de fusión, tratamiento y ajustes de composición química, que logran los estándares de calidad de las fundiciones a un costo mínimo.

\section{Referencias bibliográficas}

1) Duckworth, E., Guia a Pesquisa Operacional, Editora Atlas, São Paulo, Brasil, 1972.

2) Dos Santos-MezA, E., Dos SAntos, M.O., \& AREnAlES, M.N., A Lot-Sizing Problem in an Automated Foundry, European Journal of Operational Research, 139, 3 (2002) 490-500.

3) MAREK, ED. John WILEY \& SONS INC., Fundamental in the Production and Design of Castings, New York, 1961.

4) P. R. BEELEY, Foundry Technology, London Butterworths, 1972.

5) Raida de Jesus Silva, Reinaldo Morabito, Otimização da programação de cargas de forno em uma fábrica de fundição em aço-inox, Gestão \& Produção, vol.11 no.1 São Carlos Jan./Apr. 2004

6) R. Dan Reid, Nada R. Sanders (2005): Gestión de Operaciones. Wiley \& Sons, Inc.

7) R. W. Metzger y R. Schwarzbek, "A Linear Programming Application to Cupola Charging", The Journal of Industrial Engineering, March-Abril 1961, 87-93.

8) R.W. Hea Ne, C.R. Lopes Jr. Y P.C. Rosenthal, Principles of Metal Casting, Mc Graw-Hill, 2nd ed., 1967.

9) Text Book on Fondry Engineering-Casting Design, Nagoya International Training Centre, Nagoya, Japan, 1988.

10) Text Book on Fondry Engineering-Patterns, Nagoya International Training Centre, Nagoya, Japan, 1988.

11) Text Book on Fondry Engineering-How to Construct your Foundry, Nagoya International Training Centre, Nagoya, Japan, 1988.

\section{Consultas de internet}

a. Atlas Foundry Co. Inc. <http://www.atlas@atlasfrd.com>

b. Intercast $\mathrm{S} / \mathrm{A}$, <http://www.intercast.com.br>

3. Institute of Business Forecasters, <http://www.ibf.org>

d. São Lázaro Ind. de Injecão Ltda. <http://www.sao.lazaro@terra.com.br>

e. Sociedade Educacional Santa Catarina, <http://www.sociesc.com.br>

f. Spyro-Fundicion, <http://www.spyro.es> 